\title{
Cost of energy and mutual shadows in a two-axis tracking PV system
}

\author{
O. Perpiñán ${ }^{a}$
}

${ }^{a}$ Electrical Engineering Department, EUITI-UPM, Ronda de Valencia 3, 28012 Madrid, Spain.

\begin{abstract}
The performance improvement obtained from the use of trackers in a PV system cannot be separated from the higher requirement of land due to the mutual shadows between generators. Thus, the optimal choice of distances between trackers is a compromise between productivity and land use to minimize the cost of the energy produced by the PV system during its lifetime.

This paper develops a method for the estimation and optimization of the cost of energy function. It is built upon a set of equations to model the mutual shadows geometry and a procedure for the optimal choice of the wire cross-section. Several examples illustrate the use of the method with a particular PV system under different conditions of land and equipment costs.
\end{abstract}

This method is implemented using free software available as supplementary material.

Keywords: mutual shadows, two-axis tracking, productivity, grid-connected PV system, numerical optimization

\section{Nomenclature}

$b \quad$ Aspect ratio (length-width) of a tracker.

$B_{e f} \quad$ Effective direct irradiation (irradiance) incident on a PV generator.

$C_{E} \quad$ Cost of the energy produced by a PV system during its lifetime $(€ / \mathrm{kWh})$.

$C_{P} \quad$ Cost of the PV system including maintenance costs $(€)$.

$D_{e f} \quad$ Effective diffuse irradiation (irradiance) incident on a PV generator.

$D_{e f}^{C} \quad$ Circumsolar fraction of the effective diffuse irradiance.

$D_{e f}^{I} \quad$ Isotropic fraction of the effective diffuse irradiance.

$d_{L} \quad$ Distance between the front sides of two trackers.

$d_{W} \quad$ Distance between the short sides of two trackers.

$E_{A C} \quad$ Energy produced by a PV system during its lifetime ( $\mathrm{kWh}$ ).

$\gamma_{s} \quad$ Altitude sun angle.

Email address: o.perpinan@upm.es (O. Perpiñán) 
GCR Ground coverage ratio.

$G_{e f s}$ Effective global irradiation (irradiance) incident on a PV generator including shadows.

GRR Ground requirement ratio.

$I_{i} \quad$ Current from the i-th tracker.

$I_{i n v} \quad$ Current through the wire from the junction box to the inverter.

$k_{i}^{o} \quad$ Coefficients of the efficiency curve of a inverter

$L, W \quad$ Length and width of a tracker.

$L_{\text {ew }} \quad$ East-West distance between trackers.

$l_{e w} \quad$ East-West distance between trackers (normalized value).

$L_{i} \quad$ Length of the wires from the $\mathrm{i}$-th tracker to the junction box.

$L_{i n v} \quad$ Length of the wires from the junction box to the inverter.

$L_{n s} \quad$ North-South distance between trackers.

$l_{n s} \quad$ North-South distance between trackers (normalized value).

$P_{i n v} \quad$ Nominal power of the inverter

$p_{0} \quad$ Normalized output power of a inverter

$\psi_{s} \quad$ Azimuth sun angle.

$R_{e f} \quad$ Albedo irradiance.

$\rho \quad$ Resistivity of the wires.

$s \quad$ Shadows length.

$S_{i} \quad$ Section of the i-th tracker wires.

$S_{i n v}$ Section of the wires from the junction box to the inverter.

$\theta_{z s} \quad$ Zenith sun angle.

\section{Introduction}

The concept of a Grid Connected PV System (GCPVS) as an investment product has accelerated the development of tracking technologies. This quest for increasing the productivity of GCPVS has a cost: in general, the more precise a tracking method, the less efficient its use of land due to mutual shadows from nearby trackers.

Thus, the optimal choice of distances between trackers is a compromise between productivity and land use to minimize the cost of the energy produced by the PV system during its lifetime.

The performance of tracking PV systems and mutual shadows models have already been studied by several authors. However, nothing has yet been published integrating mutual shadows geometry, land and equipment costs, and wiring calculations, to estimate the cost of energy. 
In this context, this paper develops a method for the estimation and optimization of the cost of energy function. It is built upon a set of equations to model the mutual shadows geometry and a procedure for the optimal choice of the wire cross-section.

This document is organized as follows: the section 2 develops a set of equations for the geometry of shadows and discusses the relation between productivity, shadows and land requirement; the section 3 analyzes the wiring design issues in a two-axis tracking systems as a previous step to the cost of energy calculations; using these concepts the section 4 defines the function of the cost of the produced energy and proposes procedures for both the estimation and the minimization of this function. Finally, the section 5 illustrate the estimation and optimization of the cost function with a PV system under certain conditions of irradiation and temperature, and with several combinations of land and equipments costs.

Besides, there are three sections at the appendix with a detailed discussion of the wiring calculations (sections Appendix B and Appendix C) and costs (section Appendix A) issues.

\section{Shadows in a two-axis tracking system}

The performance of tracking PV systems and mutual shadows models have already been studied by other authors: [6] analyze the relation between shadows, area occupation, tracker and plant geometry, limitation of tracking angle and electrical configuration of the generator; [7] include a chapter devoted to geometrical considerations of tracking systems, the energy produced by each tracking technology and the analysis of mutual shadows; [8] examine the geometry of shadows in an azimuthal tracking system, applying the results to the design of a PV plant; [9] study the tracking and shading geometry for single vertical axis, single horizontal axis and two axes, and present simulation results regarding energy production and ground cover; [10] introduce an algorithm that allows the calculation of the optimal location of the PV trackers of a photovoltaic facility on a building of irregular shape, taking into account the shadows caused by the PV trackers and the obstacles that are on the building or surrounding it.

Detailed information about PV tracking, a large set of equations describing the movement of several types of trackers, and a method for mutual shadows calculation can be found in [11].

\subsection{Geometry of shadows}

The geometry of shadows in a two-axis ${ }^{1}$ tracking PV system is determined by the next parameters (figures 1 and 2):

1. The inclination of the PV generator, $\beta$, ideally equal to the zenith sun angle, $\theta_{z s}$.

2. The orientation of the PV generator, $\alpha$, ideally equal to the solar azimuth, $\psi_{s}$.

3. Aspect ratio of the tracker, $b$ : ratio between the length, $L$, and the width, $W$, of the tracker.

$$
b=\frac{L}{W}
$$

Henceforth the width $W$ will serve as the normalization factor for the distances between trackers. Unless otherwise indicated, the distances and lengths of the next sections are normalized values, using lowercase letters to denote this circumstance.

Let's define a reference coordinate system $(X, Y, Z)$ where the $X$ axis is directed towards the West, the $Y$ axis towards the South (in the north hemisphere) and the $Z$ axis towards the zenith (figure 2). Let $T_{0}$ be a tracker at the origin of coordinates, $(0,0,0)$ and let $T_{X}$ be a tracker located

\footnotetext{
${ }^{1}$ The subsequent analysis is restricted to two-axis tracking systems. The interested reader can find detailed equations of the geometry of several tracking systems in [11]
} 


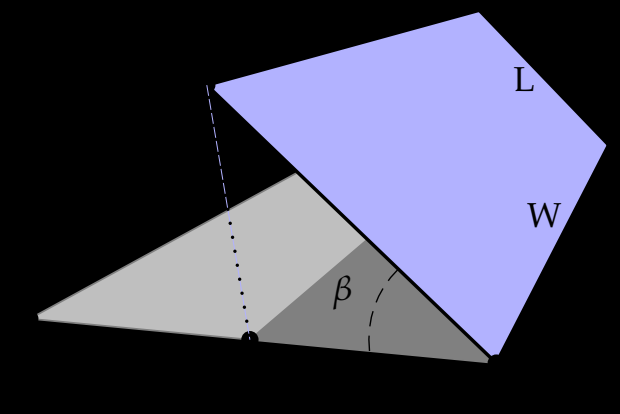

Figure 1: Dimensions of a two-axis tracker and length of its shadow.

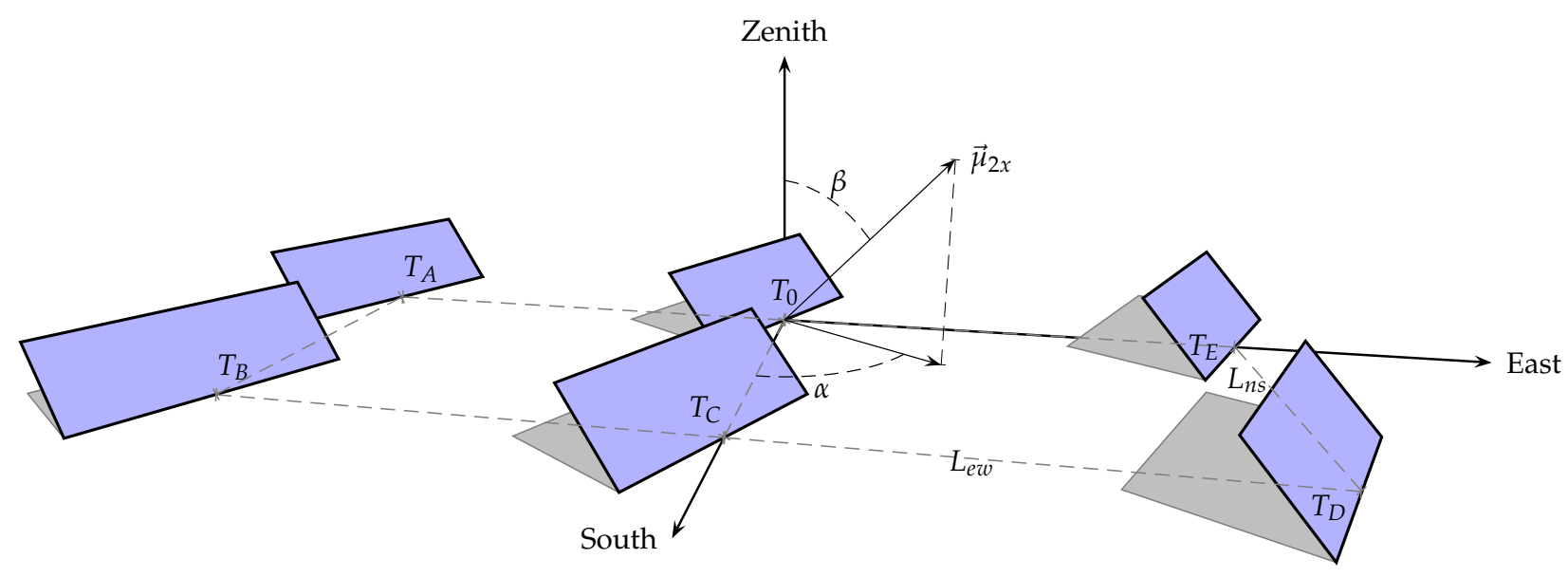

Figure 2: Mutual shadows between six two-axis trackers. 


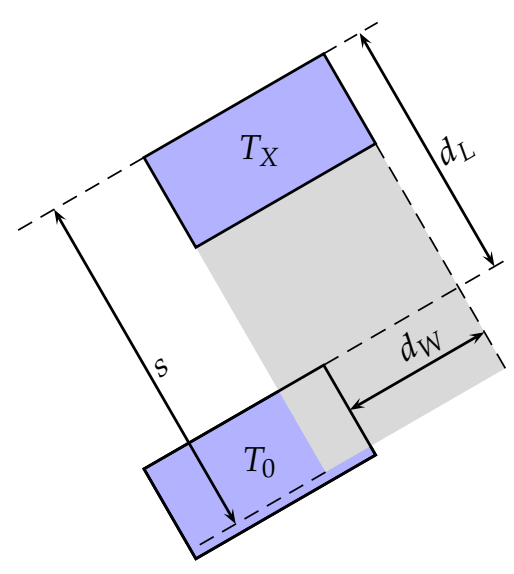

Figure 3: Distances between two trackers

at $(x, y, z)$ (figure 3). The shadows occurrence on $T_{0}$ due to $T_{X}$ is defined by the simultaneous fulfilling of three conditions:

1. $d_{W}<1$, where $d_{W}$ is the distance between the short sides of both trackers.

2. $s>d_{L}$, where $s$ is the shadows length and $d_{L}$ is the distance between the front side of both trackers.

3. The tracker $T_{X}$ is situated between the sun and the tracker $T_{0}$.

In order to check these conditions it is compulsory to compute the distances, $d_{L}$ and $d_{W}$, and the shadows length, $s$. This length is composed of two segments (figure 1):

$$
\begin{aligned}
s & =s_{1}+s_{2} \\
s_{1} & =b \cdot \cos (\beta) \\
s_{2} & =\frac{b \cdot \sin (\beta)+z}{\tan \left(\gamma_{s}\right)}
\end{aligned}
$$

where $z$ is the relative height of the tracker $T_{X}$, and $\gamma_{s}$ is the altitude sun angle. With ideal sun-tracking $\beta+\gamma_{s}=\pi / 2$, and therefore:

$$
s=\frac{b}{\cos \beta}+z \cdot \tan \beta
$$

The distances $d_{L}$ and $d_{W}$ can be easily calculated with the cross product of two vectors as distances between parallel lines. Let $r_{1}$ be a line with a director vector $\vec{u}_{r_{1}}$ and $A$ a point in this line, and $r_{2}$ a line whose vector $\vec{u}_{r_{2}}$ is parallel to $\vec{u}_{r_{1}}$, and $B$ a point located in $r_{2}$. Then, the distance between $r_{1}$ and $r_{2}$ is:

$$
d\left(r_{1}, r_{2}\right)=\frac{\left|\overrightarrow{A B} \times \vec{u}_{r_{1}}\right|}{\left|\vec{u}_{r_{1}}\right|}
$$

The distance $d_{W}$ is calculated as the distance between the lines passing through the centers of the trackers pointing at the sun. Thus, $A=(0,0,0), B=(x, y, 0)$ and $\vec{u}_{r_{1}}=\left(\sin \psi_{s}, \cos \psi_{s}, 0\right)$.

$$
d_{W}=\left|x \cos \psi_{s}-y \sin \psi_{s}\right|
$$


The distance $d_{L}$ is the distance between the lines passing through the centers of the trackers but whose direction is normal to the sun vector, $\vec{u}_{r_{1}}=\left(\cos \psi_{s},-\sin \psi_{s}, 0\right)$

$$
d_{L}=\left|x \sin \psi_{s}+y \cos \psi_{s}\right|
$$

The third condition can be checked with the sign of the scalar product $\overrightarrow{A B} \cdot \vec{u}_{r}=x \sin \psi_{s}+$ $y \cos \psi_{s}$. Thus, this condition can be stated as:

$$
x \sin \psi_{s}+y \cos \psi_{s}>0
$$

In order to save computation time, it should be noted that the left term of this equation is the right term of the equation (8) before taking absolute values.

When the three conditions apply $\left(d_{W}<1, d_{L}<s\right.$ and equation (9)), the tracker at the origin is being shaded by the tracker $T_{X}$ with a shadow factor, $S F$ :

$$
S F=\frac{\left(1-d_{W}\right) \cdot\left(s-d_{L}\right)}{b} \cdot \frac{\sin \left(\gamma_{s}\right)}{\sin \left(\gamma_{s}+\beta\right)}
$$

This shadow factor $S F$ is calculated as the ratio between the shaded area and the total area of the generator. Therefore $S F$ is zero when no shadow is received. The first fraction of this product accounts for the shadow ratio on the horizontal plane, while the second fraction projects it upon the generator plane.

The shadow factor with ideal tracking $\left(\beta+\gamma_{s}=\pi / 2\right.$, equation (5)) and coplanar trackers $(z=0)$ is:

$$
S F=\frac{\left(1-d_{W}\right) \cdot\left(s-d_{L}\right)}{S}
$$

\subsection{Shadows and productivity}

The power reduction for a given shade fraction depends on the electrical configuration of the PV system. This relation is comprised between two extremes [12]. The upper or pessimistic bound assumes that shading of any part of the PV generator produces zero output power. The lower or optimistic bound assumes that power losses due to shadows are proportional to the shaded beam radiation. Several references $[13,14,16,17,18]$ show the complexity and limits associated with the electrical models of shaded generators. Thus, the generalization of the variety of cases comprised between these two bounds is not easily feasible (although some experimental models have been already proposed [19]).

Gordon and Wenger [12] show that in a yearly basis the optimistic case is closer to a "base case" where the power of a PV module is zero when it is shaded, as an approximation to the use of bypass diodes. This approach is particularly reasonable for large generators where the effect in the global I-V characteristic of the modification of the I-V curve in some modules is lower than in small generators.

Hence, in this work a modified optimistic formulation is applied: the shadow factor proportionally reduces both the beam and the circumsolar diffuse irradiance components:

$$
G_{e f s}=D_{e f}^{I}+R_{e f}+\left(D_{e f}^{C}+B_{e f}\right) \cdot(1-S F)
$$

where $G_{e f s}$ is the global effective irradiance including shadows, $D_{e f}^{I}$ and $D_{e f}^{C}$ are, respectively, the isotropic and circumsolar fractions of the effective diffuse irradiance, $B_{e f}$ is the direct irradiance and $R_{e f}$ is the albedo irradiance.

This procedure has been sucessfully validated using performance data of a $6.02 \mathrm{MWp}$ twoaxis tracking GCPVS located in Spain [20]. However, this model imposes uncertainty in the subsequent calculation steps. Therefore, the estimation and optimization results (section 5.2 and 5.3) must be interpreted in this context. 


\subsection{The ground requirement ratio}

One of the tasks of the design of a PV tracking system is to place the set of trackers. This task must cope with the compromise of minimizing the losses due to mutual shadows while requiring the minimum land area.

A suitable approach to this problem is to simulate the planned system for a set of distances between the trackers of the plant. Without any additional constraint, the optimum design may be the one which achieves the highest productivity with the lowest ground requirement ratio. This definition of the optimization problem is not complete since the land requirements and the costs of wiring and equipments should be included as additional constraints. They are considered in section 4 .

The area of the PV generator and the total land requirement are commonly related with the Ground Coverage Ratio (GCR) [9, 8, 12]. This ratio quantifies the percentage of land being effectively occupied by the system. In order to focus on the land area required, the inverse of this ratio, the Ground Requirement Ratio (GRR), is preferable. The GRR is the ratio between the ground area required for installing the whole set of trackers and the generator area.

For this model GRR $=\frac{l_{n s} \cdot l_{e w}}{b}$. Both $l_{e w}$ and $l_{n s}$ are normalized distances, related to the absolute distances through $l_{e w}=\frac{L_{e w}}{W}$ and $l_{n s}=\frac{L_{n s}}{W}$. Therefore, $G R R=\frac{L_{e v} \cdot L_{n s}}{L \cdot W}$.

A simplified two-axis tracking PV plant can be modeled with a group of six coplanar trackers, distributed in a matrix of two rows in the North-South direction and three columns in the East-West direction (figure 2). Each tracker is named according to its position in the group. For example, a $T_{A}$ tracker could be shaded by a tracker on the East direction $\left(T_{0}\right)$ and another one in the South direction $\left(T_{B}\right)$. The model calculates the shaded irradiance for each of the six types of trackers. The average irradiance incident on a PV tracking system is the weighted average of the irradiance of the six trackers of the group. The weights are the proportion of trackers in each position.

An example will clarify this approach. In the PV plant defined in the figure A.8, there are 10 trackers $T_{0}$ (for example, the tracker no. 2 of the junction box no.1) ${ }^{2}, 2$ trackers $T_{C}$ (tracker no. 4 of the junction box 1 and tracker no. 3 of the junction box no.2), 5 trackers $T_{A}$ (for example, the tracker no.2 of the junction box 4), etc. Thus, the model will calculate six different irradiances: $G_{e f, 0}$ for the trackers $T_{0}, G_{e f, A}$ for the trackers $T_{A}$ and so on. The average irradiance of the group of 24 trackers of the figure A. 8 is:

$$
G_{e f, a v}=1 / 24 \cdot\left(10 \cdot G_{e f, 0}+5 \cdot G_{e f, A}+G_{e f, B}+2 \cdot G_{e f, C}+G_{e f, D}+5 \cdot G_{e f, E}\right)
$$

\section{Wiring design in a tracking PV system}

A grid connected PV system is electrically divided in two parts: DC (from the PV modules to the input of the inverter) and $\mathrm{AC}$ (from the output of the inverter to the grid connection point). The distances between a PV generator and its associated inverter, and between the inverter and the electrical grid are design parameters to be defined as a compromise between energy losses and wiring costs.

It is possible to show that (section Appendix B), upon a certain voltage threshold (around $475 \mathrm{~V}$ for three-phase systems), a DC distribution scheme (the inverters are situated next to the grid connection point) is better than a AC distribution scheme (the inverters are situated next to the PV generators, and the AC wiring conducts the electricity to the grid.) Due to the large sizes

\footnotetext{
${ }^{2}$ In a large PV plant there will be a high number of trackers in the $T_{0}$ location.
} 
of the tracking PV plants, the input voltage of the inverters is commonly over this threshold. Thus, the optimization procedure to be described will assume that a DC distribution scheme is adopted.

The wiring design involves the selection of a wire section adequate to the electrical current (heat dissipation) and distance (voltage losses). Let's assume that the cost of the wire is proportional to the conductor volume, and that the voltage drop to the inverter is the same from every tracker. Under such conditions, it is possible to show (section Appendix C) that the optimum sections to obtain a certain global voltage drop $\Delta U$ are calculated with (figures A.8 and C.10):

$$
\begin{aligned}
\Delta U_{i n v} & =\frac{\Delta U}{1+\sqrt{\frac{\sum_{i=1}^{n} L_{i}^{2} \cdot I_{i}}{L_{i n v}^{2} \cdot I_{i n v}}}} \\
\Delta U_{i n v} & =2 \cdot \rho \cdot \frac{L_{i n v} \cdot I_{i n v}}{S_{i n v}} \\
\Delta U_{i} & =2 \cdot \rho \cdot \frac{L_{i} \cdot I_{i}}{S_{i}}
\end{aligned}
$$

where $I_{i n v}$ is the current through the inverter wire, $I_{i}$ is the current from the i-th tracker $\left(I_{i n v}=\right.$ $\left.\sum_{i=1}^{n} I_{i}.\right), L_{i n v}$ is the length of the wires from the junction box to the inverter, $L_{i}$ is the length of the wires from the i-th tracker to the junction box, $S_{i n v}$ is the section of the inverter wires, $S_{i}$ is the section of the $\mathrm{i}$-th tracker wires and $\rho$ is the electrical resistivity of the wires.

With these equations the wire section of each part of the system can be calculated to obtain a certain value of $\Delta U$. These results have to be checked and conveniently corrected under two criteria:

- Only a set of normalized sections is available. Thus $S_{i} \in(4,6,10,16,25,35,50,70,95, \ldots) \mathrm{mm}^{2}$.

- Each type of conductor is characterized by a maximum admissible current dependent on the kind of installation. These values are commonly documented in national regulations (for example, [21] for Spain).

\section{The cost of energy}

\subsection{Definition}

The cost of the energy produced by a PV plant during its lifetime, $C_{E},(€ / \mathrm{kWh})$, is the ratio between the cost of the PV system including maintenance costs, $C_{P}(€)$, and the energy produced by a PV system during its lifetime, $E_{A C}(\mathrm{kWh})$ :

$$
C_{E}=\frac{C_{P}}{E_{A C}}
$$

$C_{P}$ can be calculated with $C_{p}=C_{c}+C_{A}+C_{P V}$, where:

- $C_{c}$ is the cost dependence on the wiring length (cable costs) modeled by a linear function $C_{c}=k_{c} \cdot L_{c}$, where the constant $k_{c}$ is the cost of cable per unit length (figure A.9) and $L_{c}$ is the length of cable.

- $C_{A}$ is the cost dependence on the required area (land costs), modeled with $C_{A}=k_{A} \cdot A_{t}$, where the constant $k_{A}$ is the cost of land per unit area, and $A_{t}$ is the total area (calculated with the distances between trackers, $L_{e w}$ and $L_{n s}$ ). 
- $C_{P V}$ is a constant term related with the cost of the PV generator, inverter, trackers and those elements whose size and quantity are not influenced by the distances between trackers (equipment costs).

$E_{A C}$ is the aggregated result of the procedure outlined in the table 1 . Since one of the steps of this procedure depends on the distances between trackers, both $E_{A C}$ and $C_{P}$ are functions of the distances $L_{E W}$ and $L_{N S}$.

\subsection{Calculation procedure}

Since this investigation is focused on the distances between trackers, the calculation procedure of the cost of energy assumes that the configuration of the PV plant has been already defined: the PV generator and inverter power; the number and type of modules, inverters and trackers; the number of modules in series and parallel; etc. With this information, the procedure is:

1. For a pair of $L_{E W}$ and $L_{N S}$ distances, the energy produced by a group of trackers with mutual shadows is calculated following the table 1 .

2. The wiring section of each circuit of the system is calculated. Both the voltage drop and the set of normalized sections constraints are applied (section 3).

3. The required land area is calculated with the distances between generators and the number of trackers included in the system (section 2.3).

4. The cost of the plant is computed as the sum of costs of wire, land and equipments (section 4.1).

5. Finally, the result is the ratio between the cost of the plant and the produced energy along its lifetime.

\subsection{Numerical optimization of the $C_{E}$ function}

Although displaying the function $C_{E}$ over a grid of distances is useful to understand its behavior (figure 4), the optimum configuration is more efficiently found with an optimization algorithm. The optimization of the distances between trackers means solving the problem in which the objective function $\left(C_{E}\right)$ is minimized by systematically choosing the values of real variables from within an allowed set.

The optimization problem is limited to a feasible region defined by several constraints:

- The separation distances must be greater than the dimensions of the trackers: $L_{E W}>W$ and $L_{N S}>L$.

- The voltage drop across a circuit must equal a predefined value (1.5\% for example).

- Only a set of wiring sections are allowed (section 3).

The iteration begins with a starting pair of distances $\left(L_{e w}, L_{n s}\right)$ in the feasible region. For this initial pair the cost of energy is calculated following the procedure outlined at section 4.2. With an optimization algorithm, a new pair of distances is selected and the calculation procedure is repeated until a convergence criterion is achieved.

For any given optimization problem, it is sensible to compare several of the available algorithms that are applicable to that problem. The performance of each algorithm strongly depends upon the problem to be solved. Moreover, not only the precision and robustness are important but also the computation speed and cost must be considered when evaluating a candidate. 


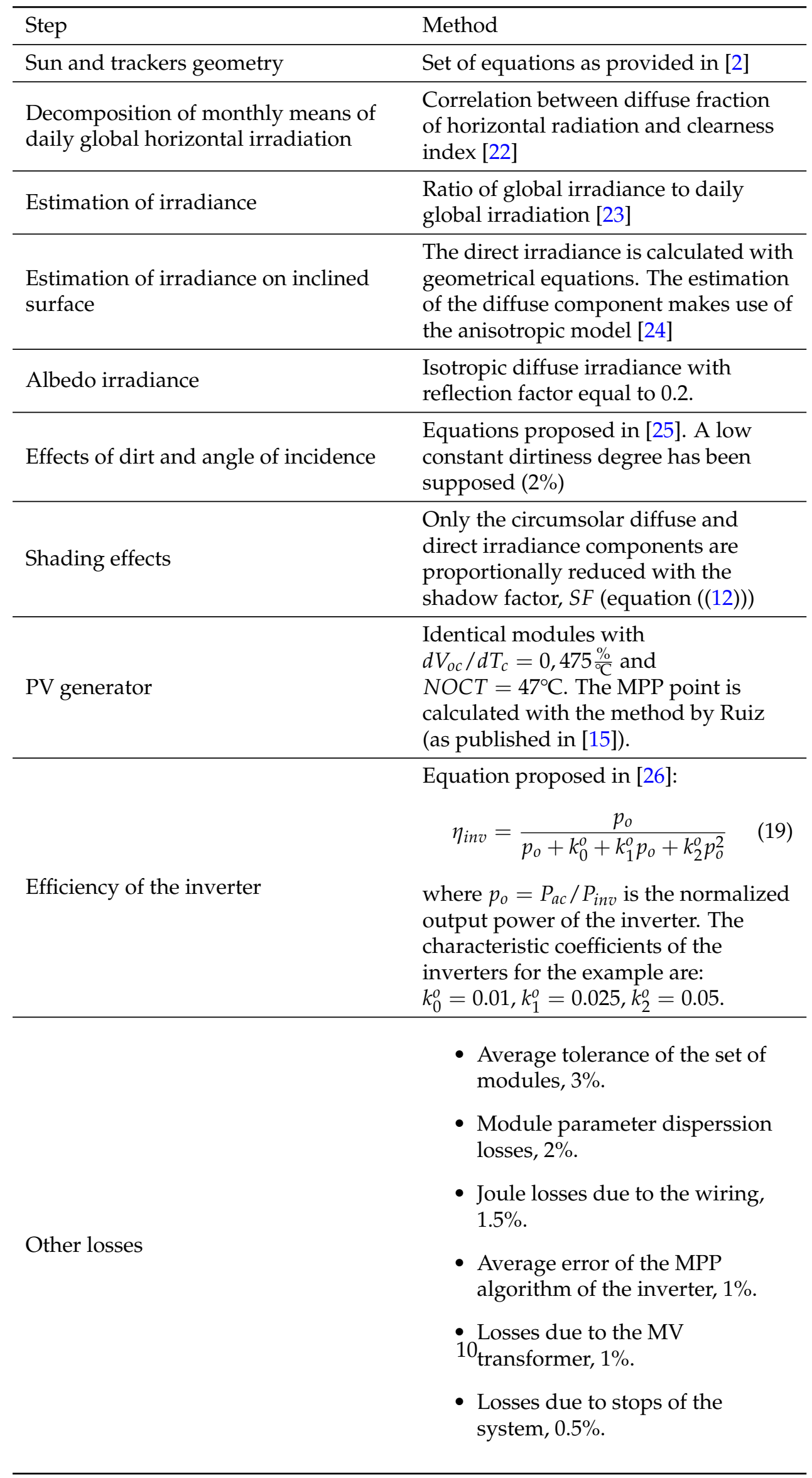

Table 1: Calculation procedure for the estimation of energy produced by a PV system from daily global horizontal irradiation data 
Although a systematic comparison of optimization algorithms is beyond the scope of this paper, two different methods have been tested: the Nelder-Mead and the COBYLA algorithms, both as implemented in the NLopt package [27].

The Nelder-Mead (or downhill simplex) method [28] is a robust and quite simple technique which approximates a local optimum of a problem with $\mathrm{N}$ variables when the objective function varies smoothly and is unimodal.

The COBYLA method (Constrained Optimization BY Linear Approximations) [29] for derivative free optimization with nonlinear inequality and equality constraints constructs successive linear approximations of the objective function and constraints via a simplex of $n+1$ points (in $\mathrm{n}$ dimensions), and optimizes these approximations in a trust region at each step.

\section{Numerical results}

The next sections illustrate the estimation (section 5.2) and optimization (section 5.3) of the $C_{E}$ function with a PV system under certain conditions of irradiation and temperature, and with several combinations of land and equipments costs. This information is related in the section Appendix A.

The use of a particular configuration for the PV system and meteorological conditions may represent a loss of generality. However, since the aim of this paper is to propose a method (and associated software) to estimate and optimize the $C_{E}$ function, the examples provide a better way to understand the development.

\subsection{Software}

The methods described in this paper have been implemented using the free software environment R [1] and several contributed packages, namely the solaR package [2] for the solar geometry, irradiation and PV energy calculations, the nloptr package for the optimization tasks, and the lattice [3], latticeExtra [4] and colorspace [5] packages as visualization tools.

The code is available at http://procomun.wordpress.com/documentos/articulos and as a supplementary material to this paper.

\subsection{Output of the $C_{E}$ function}

The figure 4 shows the output of the $C_{E}$ function for the system described in the section Appendix A over a grid of East-West and North-South distances, and for several combinations of land and PV equipment costs. The figure displays relative values of the cost (with the minima highlighted with a cross). The GRR values are superposed with gray lines.

The minima is located inside an ellipse with its major axis along the $l_{E W}$ axis. Thus, the values of the function are less sensitive to changes in the East-West distances than in the NorthSouth distances. The figure 5 shows that the minima is easily located following the curves of $l_{N S}$ (right panel) although several values of $l_{E W}$ give results very close to the minima. Therefore, the designer can choose the $L_{E W}$ distance more flexibly than the $L_{N S}$ to achieve the optimal cost of energy value.

The location of the minima moves for each combination of land and equipments costs. For the meteorological conditions of this example, it can be found near $l_{N S} \simeq 1.1$ for all the panels, but goes over the whole range of $l_{E W}$, from 1.7 for low values of equipment costs and high values of land costs, to 2.2 for the opposite combination. Besides, the minima traverse the GRR lines from $G R R \simeq 4$ for high land costs and low values of equipments costs, to $G R R \simeq 6$ for the opposite combination. 


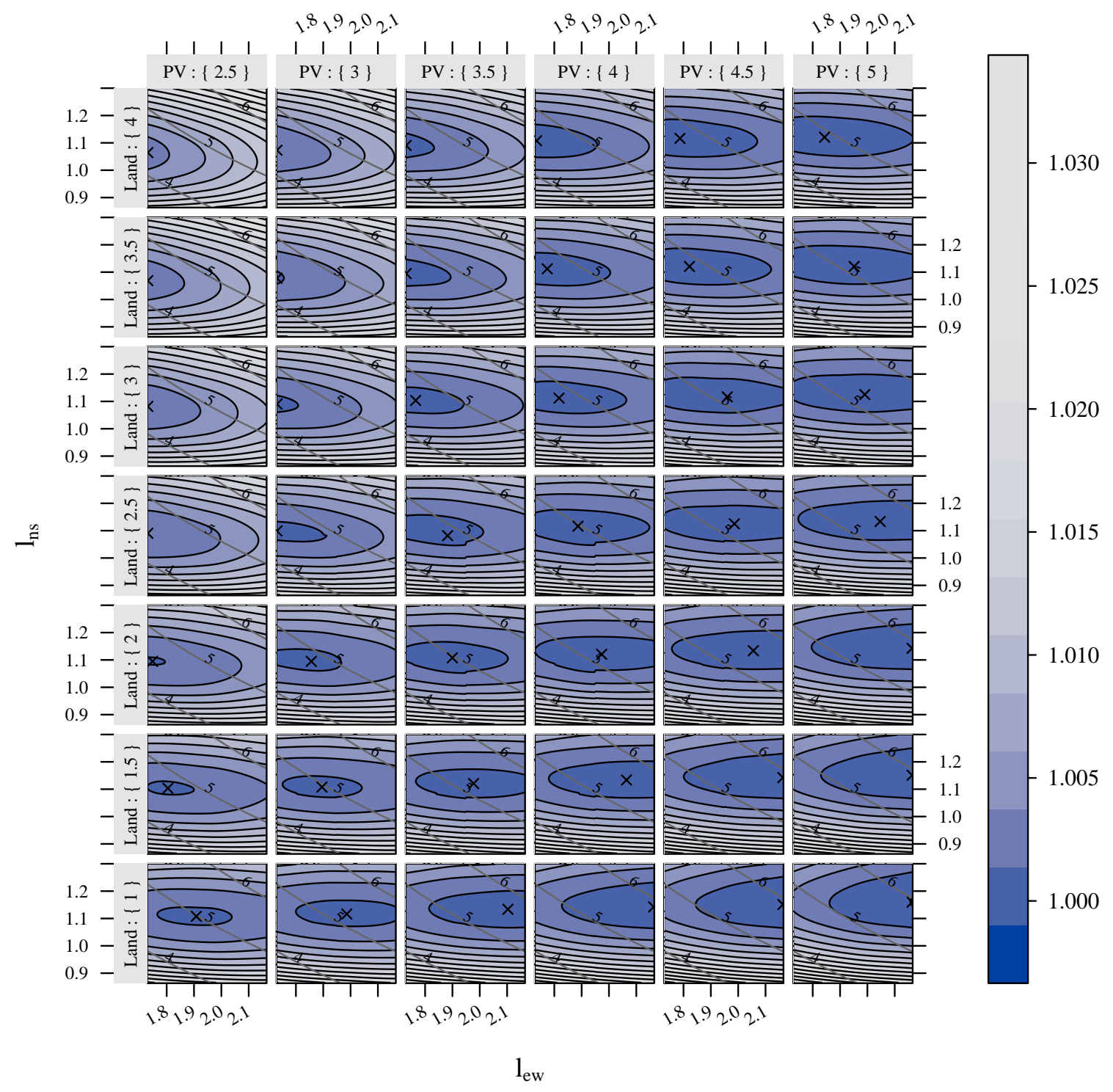

Figure 4: Cost of the energy produced by a two-axis grid-connected PV system over a grid of East-West and NorthSouth distances (normalized values), and for several combinations of cost of land per unit area $\left(€ / \mathrm{m}^{2}\right)$ and equipment costs per watt $(€ / \mathrm{Wp})$. The figure displays relative values of the cost (with the minima highlighted with a cross). The GRR values are superposed with gray lines. 


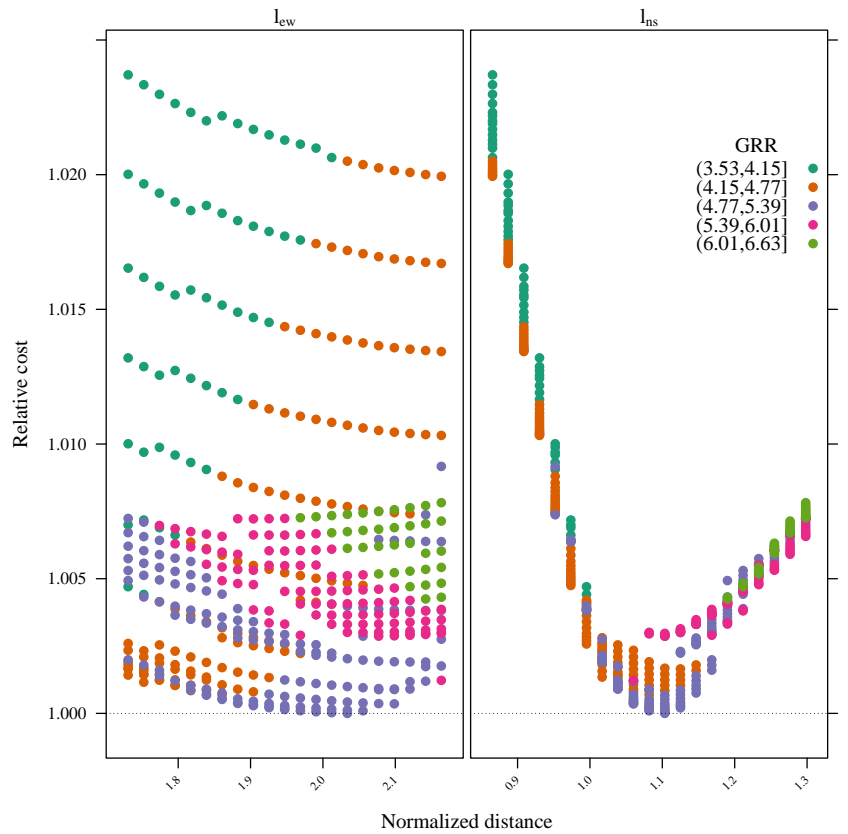

Figure 5: Subset of the figure 4 (land costs of $2 € / \mathrm{m}^{2}$ ) and equipments costs of $3,5 € / W$. The left panel shows the behavior with the North-South distance and the right panel displays the relation with the East-West distance. Each color corresponds to a GRR range.

These results must be interpreted in the context of the uncertainty limitations due to the energy estimation methods (table 1 and section 2.2). In this context, small departures from the optimum are indistinguishable.

This observation, which adds more flexibility to the design tasks, is also important when comparing the results from different optimization algorithms.

\subsection{Numerical optimization of the $C_{E}$ function}

If the equipment cost is $2,5 € / \mathrm{W}$ and the land cost is $1,5 € / \mathrm{m}^{2}$ the Nelder-Mead algorithm converges after 70 iterations with $l_{E W}=2.114\left(L_{E W}=48,853 \mathrm{~m}\right), l_{n s}=1.196\left(L_{N S}=27,63 \mathrm{~m}\right)$ and $C_{E}=7,717 \mathrm{c} € / \mathrm{kWh}$. The COBYLA algorithm converges after 35 iterations with approximately the same results.

The figures 6 (GRR values) and $7\left(l_{E W}\right.$ and $l_{N S}$, normalized distances) display the results of the COBYLA algorithm for several combinations of land and equipment costs.

The GRR values range from $G R R \simeq 6.5$ for low values of land costs and high equipment costs to $G R R \simeq 5$ for the opposite combination, with intermediate values for the rest of the grid. These results agree with the analysis of the figure 4 .

The $l_{E W}$ values (figure 7a) range from $l_{E W} \simeq 1.8$ to $l_{E W} \simeq 2.2$. The $l_{N S}$ distance (figure $7 \mathrm{~b}$ ) varies along a smaller interval: from $l_{N S} \simeq 1.12$ to $l_{E W} \simeq 1.24$. Once again this behavior was anticipated with the figures 4 and 5 . 


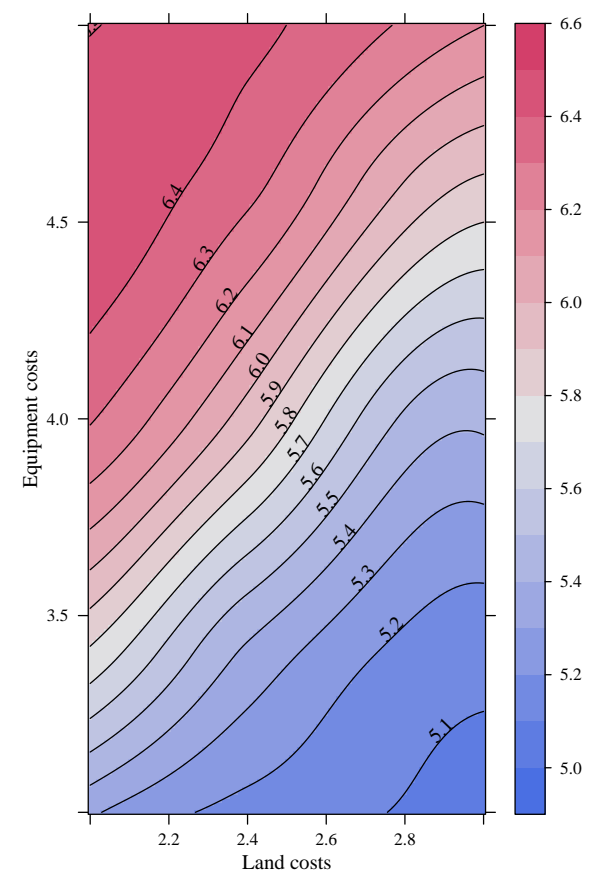

Figure 6: Results of the optimization algorithm: Ground Requirement Ratio. The horizontal axis displays the cost of land per unit area $\left(€ / \mathrm{m}^{2}\right)$ and the vertical axis shows the equipment costs per watt $(€ / \mathrm{Wp})$

\section{Conclusions}

The performance improvement obtained from the use of trackers in a PV system cannot be separated from the higher requirement of land due to the mutual shadows between generators. Thus, the optimal choice of distances between trackers is a compromise between productivity and land use to minimize the cost of the energy produced by the PV system during its lifetime.

This paper develops a method for the estimation and optimization of the cost of energy function. It is built upon a set of equations to model the mutual shadows geometry and a procedure for the optimal choice of the wire cross-section. Several examples illustrate the use of the method with a particular PV system under different conditions of land and equipment costs.

For this particular example, the minima is located inside an ellipse with its major axis along the $l_{E W}$ axis. In other words, the values of the function are less sensitive to changes in the EastWest distances than in the North-South distances. Therefore, the designer can choose the $L_{E W}$ distance more flexibly than the $L_{N S}$ to achieve the optimal cost of energy value.

The location of the minima moves for each combination of land and equipments costs. The GRR values range from $G R R \simeq 6.5$ for low values of land costs and high equipment costs to $G R R \simeq 5$ for the opposite combination, with intermediate values for the rest of the grid. The $l_{E W}$ values range from $l_{E W} \simeq 1.8$ to $l_{E W} \simeq 2.2$. The $l_{N S}$ distance varies along a smaller interval: from $l_{N S} \simeq 1.12$ to $l_{E W} \simeq 1.24$.

These results must be interpreted in the context of the uncertainty limitations due to the energy estimation methods. In this context, small departures from the optimum are indistinguishable.

This method and the examples are implemented using free software. The code is available 


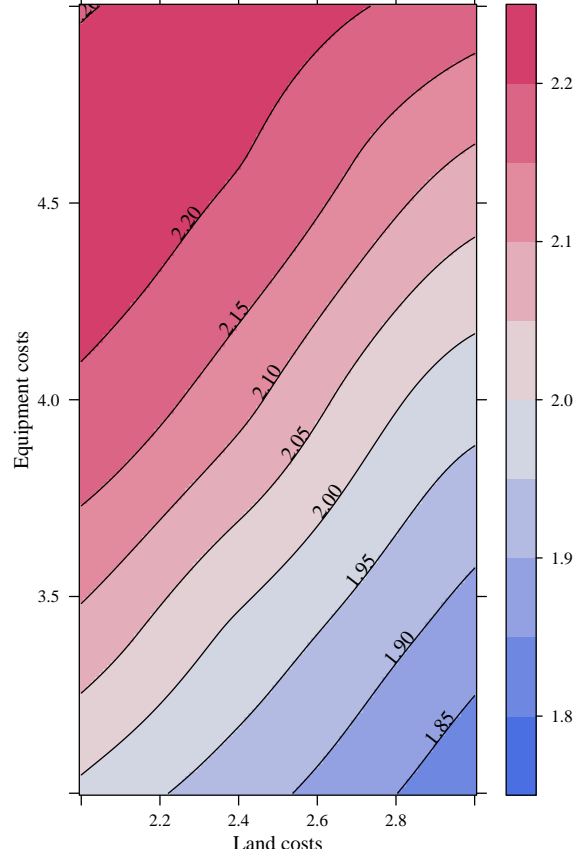

(a) $L_{e w}$

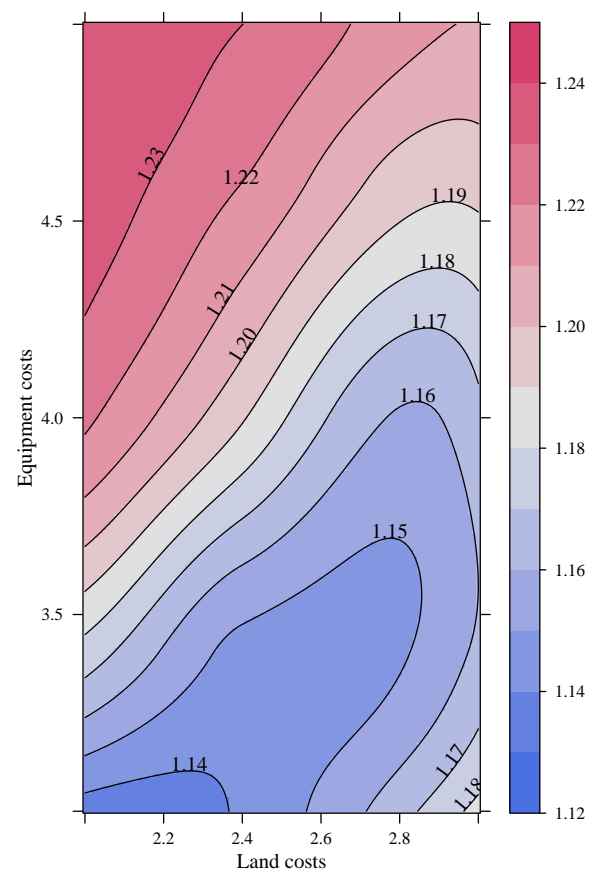

(b) $L_{n s}$

Figure 7: Results of the optimization algorithm: East-West and North-South distances (normalized values). The horizontal axis displays the cost of land per unit area $\left(€ / \mathrm{m}^{2}\right)$ and the vertical axis shows the equipment costs per watt $(€ / \mathrm{Wp})$ 
as supplementary material.

\section{Bibliography}

[1] R Development Core Team, . R: A Language and Environment for Statistical Computing. R Foundation for Statistical Computing; Vienna, Austria; 2011. ISBN 3-900051-07-0; URL http://www.R-project.org.

[2] Perpinan, O.. solaR: Calculation of Solar Radiation and PV Systems.; 2011. R package version 0.24; URL http://cran.r-project.org/web/packages/solaR/.

[3] Sarkar, D.. Lattice: Multivariate Data Visualization with R. New York: Springer; 2008. ISBN 978-0-387-75968-5; URL http: //lmdvr.r-forge.r-project .org.

[4] Sarkar, D., Andrews, F. latticeExtra: Extra Graphical Utilities Based on Lattice; 2011. URL http://R-Forge.R-project.org/projects/latticeextra/.

[5] Ihaka, R., Murrell, P., Hornik, K., Zeileis, A.. colorspace: Color Space Manipulation; 2011. R package version 1.1-0; URL http://CRAN . R-project . org/package=colorspace.

[6] Gordon, J.M., Kreider, J.F., Reeves, P.S.. Tracking and stationary flat plate solar collectors: Yearly collectible energy correlations for photovoltaics applications. Solar Energy 1991;47(4).

[7] Macagnan, M.H.. Caracterización de la radiación solar para aplicaciones fotovoltaicas en el caso de madrid. Ph.D. thesis; Instituto de Energía Solar, UPM; 1993.

[8] Lorenzo, E., Pérez, M., Ezpeleta, A., Acedo, J.. Design of tracking photovoltaic systems with a single vertical axis. Progress in Photovoltaics: Research and Applications 2002;10(8):533-543. doi: \bibinfo\{doi\}\{10.1002/pip.442\}.

[9] Narvarte, L., Lorenzo, E.. Tracking and ground cover ratio. Progress in Photovoltaics: Research and Applications 2008;16(8):703-714. doi: \bibinfo\{doi\}\{10.1002/pip.847\}.

[10] Díaz-Dorado, E., Suárez-García, A., Carrillo, C.J., Cidrás, J.. Optimal distribution for photovoltaic solar trackers to minimize power losses caused by shadows. Renewable Energy 2011;36(6):1826 - 1835. doi: \bibinfo\{doi\}\{DOI:10.1016/j.renene.2010.12.002\}.

[11] Perpiñán, O.. Energía Solar Fotovoltaica. 2011. URL http://procomun.wordpress .com/ documentos/libroesf/.

[12] Gordon, J.M., Wenger, H.J.. Central-station solar photovoltaic systems: field layout, tracker, and array geometry sensitivity studies. Solar Energy 1991;46(4):211-217.

[13] Woyte, A., Nijs, J., Belmans, R.. Partial shadowing of photovoltaic arrays with different system configurations: literature review and field test results. Solar Energy 2003;74:217233. doi: \bibinfo\{doi\}\{10.1016/S0038-092X(03)00155-5\}.

[14] Quaschning, V., Hanitsch, R.. Numerical simulation of current-voltage characteristics of photovoltaic systems with shaded solar cells. Solar Energy 1996;56(6).

[15] Alonso-García, M. C.. Caracterización y modelado de asociaciones de dispositivos fotovoltaicos. Ph.D. thesis; CIEMAT; 2005 
[16] Alonso-García, M.C., Herrmann, W., Böhmer, W., Proisy, B.. Thermal and electrical effects caused by outdoor hot-spot testing in associations of photovoltaic cells. Progress in photovoltaics: research and applications 2003;11:293-307. doi: $\backslash$ bibinfo\{doi $\}\{10.1002 /$ pip. $490\}$.

[17] Alonso-García, M.C., Ruíz, J.M., Chenlo, F.. Experimental study of mismatch and shading effects in the I-V characteristics of a photovoltaic module. Solar Energy 2006;90(3):329-340.

[18] Alonso-García, M.C., Ruíz, J.M., Herrmann, W.. Computer simulation of shading effects in photovoltaic arrays. Renewable Energy 2006;31(12):1986-1993. doi: \bibinfo\{doi\}\{10. 1016/j.renene.2005.09.030\}.

[19] Martínez-Moreno, F., Muñoz, J., Lorenzo, E.. Experimental model to estimate shading losses on PV arrays. Solar Energy Materials and Solar Cells 2010;94(12):2298 - 2303. doi: $\backslash$ bibinfo\{doi\}\{DOI:10.1016/j.solmat.2010.07.029\}.

[20] Perpiñán, O.. Statistical analysis of the performance and simulation of a two-axis tracking PV system. Solar Energy 2009;83(11):2074-2085. doi: \bibinfo\{doi\}\{10.1016/j.solener.2009. 08.008\}. URL http://oa.upm.es/1843/1/PERPINAN_ART2009_01.pdf.

[21] Ministerio de Ciencia y Tecnología, . Real Decreto 842/2002, de 2 de agosto, por el que se aprueba el Reglamento electrotécnico para baja tensión. 2002. URL http://www.ffii. nova.es/puntoinfomcyt/LegislacionSI . asp?idregl=76.

[22] Page, J.K.. The calculation of monthly mean solar radiation for horizontal and inclined surfaces from sunshine records for latitudes 40n-40s. In: U.N. Conference on New Sources of Energy; vol. 4. 1961, p. 378-390.

[23] Collares-Pereira, M., Rabl, A.. The average distribution of solar radiation: correlations between diffuse and hemispherical and between daily and hourly insolation values. Solar Energy 1979;22:155-164.

[24] Hay, J.E., McKay, D.C.. Estimating solar irradiance on inclined surfaces: A review and assessment of methodologies. Int J Solar Energy 1985;(3):203-.

[25] Martin, N., Ruíz, J.M.. Calculation of the PV modules angular losses under field conditions by means of an analytical model. Solar Energy Materials \& Solar Cells 2001;70:25-38.

[26] Jantsch, M., Schmidt, H., Schmid, J.. Results on the concerted action on power conditioning and control. In: 11th European photovoltaic Solar Energy Conference. 1992, p. $1589-1592$.

[27] Johnson, S.G.. The NLopt nonlinear-optimization package; 2011. URL http:// ab-initio.mit.edu/nlopt.

[28] Nelder, J.A., Mead, R.. A simplex method for function minimization. Computer Journal 1965;7:308-313.

[29] Powell, M.J.D.. Direct search algorithms for optimization calculations. Acta Numerica 1998;7:287-336. doi: \bibinfo\{doi\}\{10.1017/S0962492900002841\}. http:// journals.cambridge.org/article_S0962492900002841; URL http://dx.doi.org/10. 1017/S0962492900002841. 


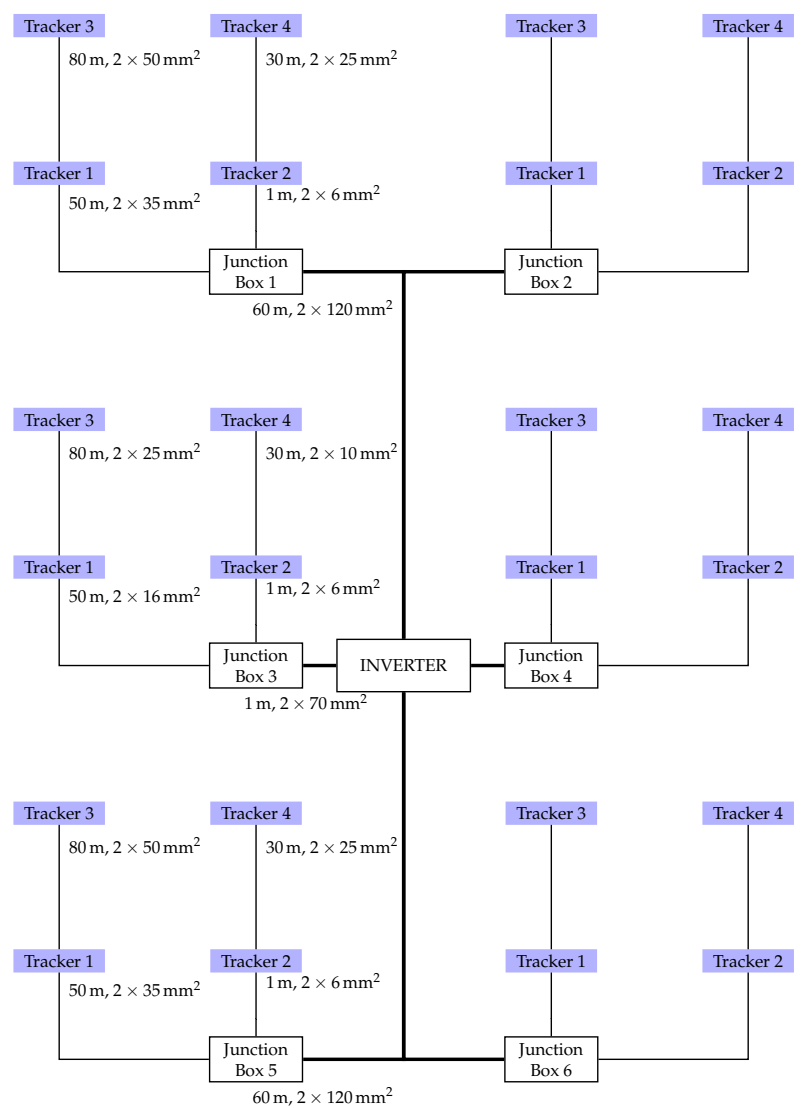

Figure A.8: Layout of the PV system. For illustrative purposes, the wires are labeled with the values of lengths and cross-sections corresponding to $L_{E W}=50 \mathrm{~m}$ and $L_{N S}=30 \mathrm{~m}$.

[30] Perpiñán, O., Lorenzo, E., Castro, M.A., Eyras, R.. Energy payback time of grid connected PV systems: comparison between tracking and fixed systems. Progress in Photovoltaics: Research and Applications 2009;17:137-147. doi: \bibinfo\{doi\}\{10.1002/pip.871\}.

\section{Appendix A. Data for the examples}

This section provides information of the PV system, land and equipment costs, irradiation and temperature conditions, used in part of the paper to illustrate the definition of the $C_{E}$ function in the section 5 .

The PV system is composed of 24 two-axis trackers. Each of the trackers $(L=9,8 \mathrm{~m}, W=$ 23,11 m) supports a PV array of 132 modules of $200 \mathrm{Wp}$ with 12 modules in series and 11 in parallel. Detailed information about this system can be found in a previous paper [30].

A group of four trackers forms a PV generator with a nominal power of 105,6 kWp. Each group feeds one of a set of six inverters of $100 \mathrm{kWp}$. The inverters are hosted in a building approximately at the geometrical center of the plant (figure A.8)

This system is simulated with the meteorological conditions of a site located at Sevilla (latitude $\phi=37.2^{\circ}$ ) (table Appendix A) 


\begin{tabular}{lllllllllllll}
\hline Parameter & Jan & Feb & Mar & Apr & May & Jun & Jul & Aug & Sep & Oct & Nov & Dec \\
\hline$G_{d m}(0)\left(\mathrm{Wh} / \mathrm{m}^{2}\right)$ & 2766 & 3491 & 4494 & 5912 & 6989 & 7742 & 7919 & 7027 & 5369 & 3562 & 2814 & 2179 \\
\hline$T_{a}\left({ }^{\circ} \mathrm{C}\right)$ & 10 & 14.1 & 15.6 & 17.2 & 19.3 & 21.2 & 28.4 & 29.9 & 24.3 & 18.2 & 17.2 & 15.2 \\
\hline
\end{tabular}

Table A.2: Meteorological data (monthly means of global irradiation and ambient temperature) of Sevilla, Spain.

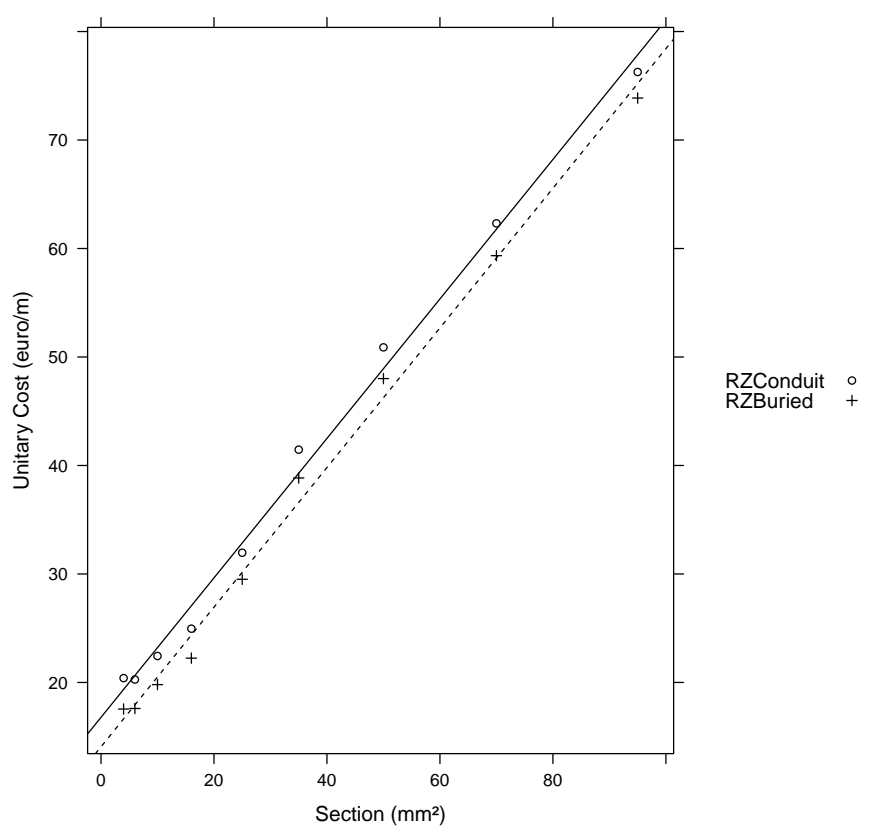

Figure A.9: Unitary costs for different sections of free-halogen cable (from the PREOC database).

The information for the cable costs have been extracted from the PREOC database. This database is available online ${ }^{3}$ and it is a frequently used reference for industrial and civil works prices in Spain. The figure A.9 displays the unitary costs for a free-halogen cable either buried or with conduits.

The equipment cost range (from $2,5 € / \mathrm{W}$ to $5 € / \mathrm{W}$ ) is inspired from the SolarBuzz webpage ${ }^{4}$ while the land cost range (from $1,5 € / \mathrm{m}^{2}$ to $\left.4 € / \mathrm{m}^{2}\right)^{5}$ is derived from several professional forums ${ }^{6}$.

\section{Appendix B. AC versus DC}

Let's show that, upon a certain voltage threshold, a DC distribution scheme (the inverters are situated next to the grid connection point) is better than a AC distribution scheme (the inverters

\footnotetext{
${ }^{3}$ http: //www.preoc.es/

${ }^{4}$ http://solarbuzz.com/facts-and-figures/retail-price-environment

${ }^{5}$ For example, $2,5 € / \mathrm{m}^{2}$ is the result of a yearly land rental of $1000 €$ per hectare during 25 years.

${ }^{6}$ For example http: //www. solarweb.net.
} 
are situated next to the PV generators, and the AC wiring conducts the electricity to the grid.)

Some assumptions are needed for a clearer proof:

1. The connection to the distribution grid is three-phase through three-phase inverters without neutral wire.

2. The efficiency of the inverter is almost constant with a value of 0.95 .

3. The reactive power of the inverter is negligible.

4. The admissible voltage drop is $\Delta V=1.5 \% \cdot V_{\text {nom }}$, where $V_{\text {nom }}$ is the nominal voltage for each part. For the AC part is $V_{\text {nom }}=400 \mathrm{~V}$.

5. Both distribution schemes use a wire with the same conductivity, $\sigma$.

The intersection point is found when both distribution schemes require the same mass of wire:

$$
2 \cdot S_{d c} \cdot L_{w}=3 \cdot S_{3 a c} \cdot L_{w}
$$

where $L_{w}$ is the wire length, $S_{d c}$ is the section for the DC distribution scheme and $S_{3 a c}$ is the section for the AC distribution scheme.

The DC section is calculated with:

$$
S_{d c}=\frac{2 \cdot L_{w} \cdot I_{d c}}{\sigma \cdot \Delta V_{d c}}
$$

and the AC section is calculated with:

$$
S_{3 a c}=\frac{\sqrt{3} \cdot L_{w} \cdot I_{3 a c}}{\sigma \cdot \Delta V_{3 a c}}
$$

where the assumptions no. 3 and no. 5 have been considered.

These equations can be rewritten with the relations between electrical power, voltage and current (assumption no.4):

$$
\begin{aligned}
S_{d c} & =\frac{2 \cdot L_{w} \cdot P_{d c}}{\sigma \cdot 1.5 \% \cdot V_{d c}^{2}} \\
S_{3 a c} & =\frac{L_{w} \cdot P_{a c}}{\sigma \cdot 1.5 \% \cdot V_{a c}^{2}}
\end{aligned}
$$

Equation (B.1) is now:

$$
2 \cdot \frac{2 \cdot L_{w} \cdot P_{d c}}{\sigma \cdot 1.5 \% \cdot V_{d c}^{2}}=3 \cdot \frac{L_{w} \cdot P_{a c}}{\sigma \cdot 1.5 \% \cdot V_{a c}^{2}}
$$

It can be simplified using the assumption no.2:

$$
\frac{4}{V_{d c}^{2}}=\frac{3 \cdot \eta_{i n v}}{V_{a c}^{2}}
$$

which leads to the final result (with $V_{a c}=400 \mathrm{~V}$ and $\eta_{i n v}=0.95$ ):

$$
V_{d c} \simeq 473 \mathrm{~V}
$$

Thus, if the average $V_{m p p}$ voltage of the PV generator is above $473 \mathrm{~V}$, the DC distribution scheme is preferable to the $\mathrm{AC}$ distribution scheme to optimize the total mass of wire. 


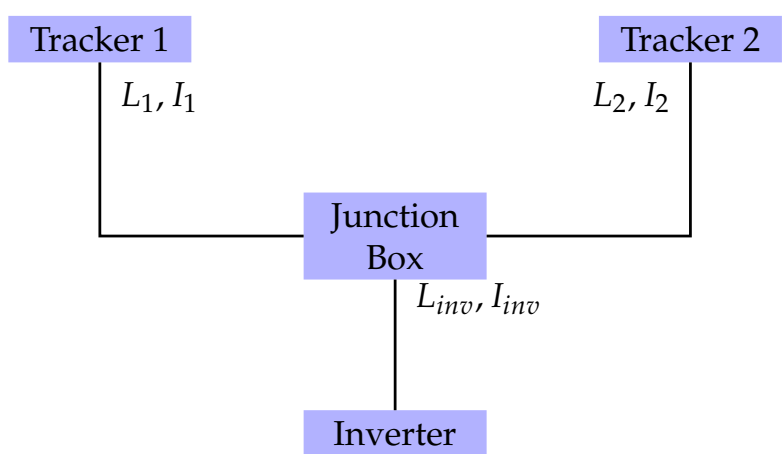

Figure C.10: Wire lengths and currents in a PV system with two trackers feeding a common inverter through a junction box.

\section{Appendix C. Wire section optimization}

In order to calculate the wire section for each part of the system, two assumptions are needed:

- The cost of the wire is proportional to the conductor volume.

- The voltage drop to the inverter is the same from every tracker.

For two generators feeding a common inverter (figure C.10), the voltage drops are:

$$
\begin{aligned}
& \Delta U_{i n v}+\Delta U_{1}=\Delta U \\
& \Delta U_{i n v}+\Delta U_{2}=\Delta U
\end{aligned}
$$

where $\Delta U_{i n v}$ stands for the voltage drop from the junction box to the inverter, $\Delta U_{i}$ stands for the voltage drop from the tracker $i$ to the junction box, and $\Delta U$ is the voltage drop from a tracker to the inverter. These voltage drops can be calculated with:

$$
\begin{aligned}
\Delta U_{i n v} & =2 \cdot \rho \cdot \frac{L_{i n v} \cdot I_{i n v}}{S_{i n v}} \\
\Delta U_{i} & =2 \cdot \rho \cdot \frac{L_{i} \cdot I_{i}}{S_{i}}
\end{aligned}
$$

where $I_{i n v}$ is the current through the inverter wire, $I_{i}$ is the current from the i-th tracker, $L_{i n v}$ is the length of the wires from the junction box to the inverter, $L_{i}$ is the length of the wires from the i-th tracker to the junction box, $S_{i n v}$ is the section of the wires from the junction box to the inverter, $S_{i}$ is the section of the $\mathrm{i}$-th tracker wires and $\rho$ is the electrical resistivity of the wires.

Of course, $I_{i n v}=I_{1}+I_{2}$. The total wire volume, assuming a DC distribution schema (section Appendix B), is:

$$
\begin{aligned}
V o l & =2 \cdot L_{i n v} \cdot S_{i n v}+2 \cdot L_{1} \cdot S_{1}+2 \cdot L_{2} \cdot S_{2} \\
& =4 \cdot \rho \cdot\left(\frac{L_{i n v}^{2} \cdot I_{i n v}}{\Delta U_{i n v}}+\frac{L_{1}^{2} \cdot I_{1}}{\Delta U_{1}}+\frac{L_{2}^{2} \cdot I_{2}}{\Delta U_{2}}\right)
\end{aligned}
$$

With the equations (C.1) and (C.2) the wire volume is:

$$
V o l=4 \cdot \rho \cdot\left(\frac{L_{i n v}^{2} \cdot I_{i n v}}{\Delta U_{i n v}}+\frac{L_{1}^{2} \cdot I_{1}}{\Delta U-\Delta U_{i n v}}+\frac{L_{2}^{2} \cdot I_{2}}{\Delta U-\Delta U_{i n v}}\right)
$$


The optimum choice of sections is the result of $\frac{d V o l}{d U}=0$.

$$
\frac{L_{i n v}^{2} \cdot I_{i n v}}{\Delta U_{i n v}}=\frac{L_{1}^{2} \cdot I_{1}+L_{2}^{2} \cdot I_{2}}{\Delta U-\Delta U_{i n v}}
$$

and, therefore,

$$
\frac{\Delta U-\Delta U_{i n v}}{\Delta U_{i n v}}=\frac{L_{1}^{2} \cdot I_{1}+L_{2}^{2} \cdot I_{2}}{L_{i n v}^{2} \cdot I_{i n v}}
$$

which can be rewritten to:

$$
\Delta U_{i n v}=\frac{\Delta U}{1+\sqrt{\frac{L_{1}^{2} \cdot I_{1}+L_{2}^{2} \cdot I_{2}}{L_{i n v}^{2} \cdot I_{i n v}}}}
$$

This last equation can be generalized for a higher number of trackers:

$$
\Delta U_{i n v}=\frac{\Delta U}{1+\sqrt{\frac{\sum_{i=1}^{n} L_{i}^{2} \cdot I_{i}}{L_{i n v}^{2} \cdot I_{i n v}}}}
$$

where $I_{i n v}=\sum_{i=1}^{n} I_{i}$.

With the equation (C.1) to (C.4) and (C.11), the wire section of each part of the system can be calculated to obtain a certain value of $\Delta U$. These results have to be checked and conveniently corrected under two criteria:

- Only a set of normalized sections is available. Thus $S_{i} \in(4,6,10,16,25,35,50,70,95, \ldots) \mathrm{mm}^{2}$.

- Each type of conductor is characterized by a maximum admissible current dependent on the kind of installation. These values are commonly documented in national regulations (for example, [21] for Spain). 